Article

\title{
On a New Result on the Ratio Exponentiated General Family of Distributions with Applications
}

\author{
Rashad A. R. Bantan ${ }^{1}$, Farrukh Jamal ${ }^{2} \mathbb{(}$, Christophe Chesneau ${ }^{3, *}$ and Mohammed Elgarhy ${ }^{4}$ \\ 1 Department of Marine Geology, Faculty of Marine Sience, King AbdulAziz University, \\ Jeddah 21551, Saudi Arabia; rbantan@kau.edu.sa \\ 2 Department of Statistics, Govt. S.A Postgraduate College Dera Nawab Sahib, Bahawalpur, \\ Punjab 63100, Pakistan; drfarrukh1982@gmail.com \\ 3 Department of Mathematics, Université de Caen, LMNO, Campus II, Science 3, 14032 Caen, France \\ 4 Valley High Institute for Management Finance and Information Systems, Obour, Qaliubia 11828, Egypt; \\ m_elgarhy85@sva.edu.eg \\ * Correspondence: christophe.chesneau@unicaen.fr; Tel.: +33-02-3156-7424
}

Received: 21 March 2020; Accepted: 14 April 2020; Published: 15 April 2020

\begin{abstract}
In this paper, we first show a new probability result which can be concisely formulated as follows: the function $2 G^{\beta} /\left(1+G^{\alpha}\right)$, where $G$ denotes a baseline cumulative distribution function of a continuous distribution, can have the properties of a cumulative distribution function beyond the standard assumptions on $\alpha$ and $\beta$ (possibly different and negative, among others). Then, we provide a complete mathematical treatment of the corresponding family of distributions, called the ratio exponentiated general family. To link it with the existing literature, it constitutes a natural extension of the type II half logistic-G family or, from another point of view, a compromise between the so-called exponentiated-G and Marshall-Olkin-G families. We show that it possesses tractable probability functions, desirable stochastic ordering properties and simple analytical expressions for the moments, among others. Also, it reaches high levels of flexibility in a wide statistical sense, mainly thanks to the wide ranges of possible values for $\alpha$ and $\beta$ and thus, can be used quite effectively for the real data analysis. We illustrate this last point by considering the Weibull distribution as baseline and three practical data sets, with estimation of the model parameters by the maximum likelihood method.
\end{abstract}

Keywords: exponentiated-G family; Marshall-Olkin-G family; stochastic ordering; maximum likelihood estimation; data analysis

MSC: 60E05; 62E15; 62F10

\section{Introduction}

The sharp and fine analysis of modern data sets often requires in-depth statistical treatments, beyond the capabilities of the usual statistical models. That is, particular attentions was made to define models with new features in this regard, motivating the development of general families of distributions having the ability to generate flexible distributions. Among these families, we may mention the skew-normal family pioneered by [1], the exponentiated-generated (Exp-G) family proposed by [2], the Marshall-Olkin-generated (MO-G) family studied by [3], the order statistics-generated family introduced by [4], the Sinh-arcsinh-generated family developed by [5], the beta-generated (B-G) family introduced by [6], the transmuted-generated (T-G) family developed by [7], the gamma-generated (Gam-G) family proposed by [8] and the Pareto ArcTan family developed by [9]. Each of them has generated a plethora of distributions and statistical models, widely used in practice. 
In order to illustrate their diversity, we now succinctly present some of these families from the mathematical point of view. The most simple one uses the power transform; the Exp-G family is defined by the following cumulative distribution function (cdf): $F(x ; \alpha, \xi)=G(x ; \xi)^{\alpha}, x \in \mathbb{R}$, where $G(x ; \xi)$ denotes a cdf of a continuous distribution, with $\xi$ as a generic parameters vector, and $\alpha>0$. Based on the geometric series expansion as a prime structure, the MO-G is defined by the following cdf: $F(x ; \theta, \xi)=1-\theta(1-G(x ; \xi)) /[1-(1-\theta)(1-G(x ; \xi))], x \in \mathbb{R}$, where $\theta>$ 0 . Centered around the so-called beta function, the B-G family is defined by the following cdf: $F(x ; \alpha, \beta, \xi)=B_{G(x ; \xi)}(\alpha, \beta) / B_{1}(\alpha, \beta), x \in \mathbb{R}$, where $B_{y}(\alpha, \beta)=\int_{0}^{y} t^{\alpha-1}(1-t)^{\beta-1} d t$, and $\alpha, \beta>0$. By the use of the quadratic rank transmutation, the T-G family is defined by the following cdf: $F(x ; \lambda, \xi)=$ $G(x ; \xi)[(1+\lambda)-\lambda G(x ; \xi)], x \in \mathbb{R}$, with $\lambda \in[-1,1]$. Finally, emerging from the so-called gamma function, the Gam-G family is defined by the following cdf: $F(x ; \alpha, \xi)=\gamma(\alpha,-\log [1-G(x ; \xi)]) / \Gamma(\alpha)$, where $\gamma(\alpha, y)=\int_{0}^{y} t^{\alpha-1} e^{-t} d t, \Gamma(\alpha)=\lim _{y \rightarrow+\infty} \gamma(\alpha, y)$ and $\alpha>0$.

In this paper, we contribute to the subject by going further some standard assumptions; we first show that the following function:

$$
F(x ; \alpha, \beta, \xi)=\frac{2 G(x ; \xi)^{\beta}}{1+G(x ; \xi)^{\alpha}}, \quad x \in \mathbb{R}
$$

remains a valid cdf under wide assumptions on the parameters $\alpha$ and $\beta$ (allowing possible different and/or negative values). This cdf can be viewed as an extension of the type II half logistic-G (TIIHL-G) family by [10] or, alternatively, an hybrid version of the cdfs corresponding to the Exp-G and MO-G families. More specifically, if $\alpha=0$, it corresponds to the cdf of the Exp-G family, if $\beta=\alpha=1$, it corresponds to the cdf of the MO-G family with parameter $\theta=1 / 2$ (also corresponding to the M-G family by [11]) and, if $\beta=\alpha$, it becomes the cdf of the type II half logistic-G (TIIHL-G) family. However, to the best of our knowledge, the general case including possible $\beta \neq \alpha$ remains unexplored, and is the motor of this study.

We thus introduced the ratio exponentiated general (or generated) (RE-G) family of distributions defined by the cdf (1), with values of $\alpha$ and $\beta$ to be specified later. We investigate some interesting mathematical properties of the family, including the analytical expressions the main corresponding functions, useful stochastic ordering results, analysis of the asymptotes and mode(s) for the probability density and hazard rate functions, series expansions of the probability density function, various measures involving moments and general formula related to the maximum likelihood method. Then, we pay a particular attention on a special member of the family based on the Weibull distribution as baseline. It constitutes a new and simple four-parameters distribution with many attractive features for the statistician. In particular, we show that the corresponding probability density and hazard rate functions enjoy flexible shape properties, which are desirable for modelling purposes. Thus, the related model is able to capture the complexity of various kinds of data. We illustrate this claim by the use of the maximum likelihood method (validated by a simulation study), and the means of three different practical sets. Our model reveals to be competitive in comparison to other five strong model competitors, with notable gain in terms of well-established criteria.

This paper is organized as follows. In Section 2, we present the mathematical foundations of the RE-G family. Some notable properties are derived in Section 3. Numerical studies are provided in Section 4, including a golden member of the RE-G family, simulation study and analyses of three practical data sets, with discussions. Section 5 draws some concluding remarks.

\section{The RE-G Family}

Here, we give the essential of the RE-G family, including its genesis and the main corresponding functions. 


\subsection{Central Result}

The RE-G family is based on the following new theoretical result.

Theorem 1. Let $\alpha, \beta \in \mathbb{R}, G(x ; \xi)$ be the cdf of a continuous distribution and $F(x ; \alpha, \beta, \xi)$ be the function defined by

$$
F(x ; \alpha, \beta, \xi)=\frac{2 G(x ; \xi)^{\beta}}{1+G(x ; \xi)^{\alpha}}, \quad x \in \mathbb{R} .
$$

Then, $F(x ; \alpha, \beta, \xi)$ has the properties of a cdf if $\beta \neq 0$ and $\beta \geq \alpha / 2$, or if $\beta=0$ and $\alpha<0$.

Proof. Hereafter, for the sake conciseness, we set " $\lim _{x \rightarrow-\infty}$ " for " $G(x ; \xi) \rightarrow 0$ ", and " $\lim _{x \rightarrow+\infty}$ " for " $G(x ; \xi) \rightarrow 1$ ". Now, let us distinguish the three following (non overlapped) cases: $\beta>0$ on the one hand, then $\beta<0$ and $\beta=0$ on the other hand.

Case I: $\beta>0$. As composition of continuous functions, $F(x ; \alpha, \beta, \xi)$ is a continuous function. For any $\alpha \in \mathbb{R}$, we have $\lim _{x \rightarrow+\infty} F(x ; \alpha, \beta, \xi)=1$. Let us now investigate $\lim _{x \rightarrow-\infty} F(x ; \alpha, \beta, \xi)$ according to the cases $\alpha>0, \alpha=0$ and $\alpha \leq 0$.

If $\alpha>0$, we have $F(x ; \alpha, \beta, \xi) \sim 2 G(x ; \xi)^{\beta}$ and $\lim _{x \rightarrow-\infty} F(x ; \alpha, \beta, \xi)=0$. If $\alpha=0$, we have $F(x ; \alpha, \beta, \xi)=G(x ; \xi)^{\beta}$ and $\lim _{x \rightarrow-\infty} F(x ; \alpha, \beta, \xi)=0$. If $\alpha<0$, we have $F(x ; \alpha, \beta, \xi) \sim$ $2 G(x ; \xi)^{\beta-\alpha}$ and, since $\beta>0>\alpha$, we have $\lim _{x \rightarrow-\infty} F(x ; \alpha, \beta, \xi)=0$.

Now, by denoting $g(x ; \xi)$ the pdf corresponding to $G(x ; \xi)$, upon differentiation with respect to $x$, we have, almost everywhere,

$$
F^{\prime}(x ; \alpha, \beta, \xi)=\frac{2 G(x ; \xi)^{\beta-1} g(x ; \xi)\left[\beta+(\beta-\alpha) G(x ; \xi)^{\alpha}\right]}{\left[1+G(x ; \xi)^{\alpha}\right]^{2}}
$$

Let us now distinguish the cases $\beta-\alpha \geq 0$ and $\beta-\alpha<0$. If $\beta-\alpha \geq 0$, since $\beta>0$ and $G(x ; \xi)^{\alpha} \geq 0$, we have $\beta+(\beta-\alpha) G(x ; \xi)^{\alpha} \geq 0$, implying that $F^{\prime}(x ; \alpha, \beta, \xi) \geq 0$. If $\beta-\alpha<0$, since $\beta>0$ and $G(x ; \xi)^{\alpha} \in(0,1)$, we have $\beta+(\beta-\alpha) G(x ; \xi)^{\alpha} \geq \beta+(\beta-\alpha)=2 \beta-\alpha$, which is positive if $\beta \geq \alpha / 2$, implying that $F^{\prime}(x ; \alpha, \beta, \xi) \geq 0$. Thus, if $\beta \geq \alpha / 2, F(x ; \alpha, \beta, \xi)$ is increasing. By putting all the above results together, we prove that $F(x ; \alpha, \beta, \xi)$ has the properties of a cdf.

Case II: $\beta<0$. For this case, we can express $F(x ; \alpha, \beta, \xi)$ as

$$
F(x ; \alpha, \beta, \xi)=\frac{G(x ; \xi)^{-\alpha}}{G(x ; \xi)^{-\alpha}} \times \frac{2 G(x ; \xi)^{\beta}}{1+G(x ; \xi)^{\alpha}}=\frac{2 G(x ; \xi)^{\theta}}{1+G(x ; \xi)^{\gamma}}
$$

where $\theta=\beta-\alpha$ and $\gamma=-\alpha$. Let us now remark that, if $\beta \geq \alpha / 2$, then we have $\alpha<0$, implying that $\alpha / 2>\alpha$ and, as consequence, $\theta>0$. Thus, we can follow the arguments developed for Case I, but with $\theta$ and $\gamma$ instead of $\beta$ and $\alpha$, respectively; $F(x ; \alpha, \beta, \xi)$ has the properties of a cdf if $\theta \geq \gamma / 2$. We end the proof of this case by noticing that $\theta \geq \gamma / 2$ is equivalent to $\beta-\alpha \geq-\alpha / 2$, hence $\beta \geq \alpha / 2$.

Case III: $\beta=0$. The case $\alpha \geq 0$ is directly excluded because $\lim _{x \rightarrow-\infty} F(x ; \alpha, \beta, \xi)=2 \neq 0$. Now, if $\alpha<0$, then we can write

$$
F(x ; \alpha, 0, \xi)=\frac{G(x ; \xi)^{-\alpha}}{G(x ; \xi)^{-\alpha}} \times \frac{2}{1+G(x ; \xi)^{\alpha}}=\frac{2 G(x ; \xi)^{\theta}}{1+G(x ; \xi)^{\gamma}}
$$

with $\theta=-\alpha$ and $\gamma=-\alpha$. Since $\theta>0$, owing to the same arguments used for Case I, $F(x ; \alpha, 0, \xi)$ is a cdf if $\theta \geq \gamma / 2$, hence $\alpha / 2 \geq \alpha$, which is always satisfied in this case.

The proof of Theorem 1 is ended. 
We claim that Theorem 1 provides a contribution to the TIIHL-G family proposed by [10]. Indeed, we recall that the TIIHL-G family is defined with the $\operatorname{cdf} F(x ; \alpha, \beta, \xi)$ with $\beta=\alpha$ only; Theorem 1 shows that it can be significantly extended and enriched with wider ranges of values for $\alpha$ and/or $\beta$, allowing negative values for $\alpha$ and $\beta$ as well. To the best of our knowledge, such a result is unexplored in the literature and opens new perspectives of work. The next sections are devoted to the prime theoretical or practical features of the RE-G family.

\subsection{Definition and Main Functions}

The definition of the RE-G family, as well as some important functions, are described below. Based on Theorem 1, we define the RE-G family by the cdf given by

$$
F(x ; \alpha, \beta, \xi)=\frac{2 G(x ; \xi)^{\beta}}{1+G(x ; \xi)^{\alpha}}, \quad x \in \mathbb{R}
$$

where $\alpha$ and $\beta$ are two shapes parameters satisfying $\beta \neq 0$ and $\beta \geq \alpha / 2$, or $\beta=0$ and $\alpha<0$ (these assumptions will hold implicitly in the sequel of the study). Upon differentiation of $F(x ; \alpha, \beta, \xi)$ with respect to $x$ (almost everywhere), the corresponding probability density function (pdf) is obtained as

$$
f(x ; \alpha, \beta, \xi)=\frac{2 G(x ; \xi)^{\beta-1} g(x ; \xi)\left[\beta+(\beta-\alpha) G(x ; \xi)^{\alpha}\right]}{\left[1+G(x ; \xi)^{\alpha}\right]^{2}}, \quad x \in \mathbb{R} .
$$

This pdf will be central to express important measures and functions of the RE-G family (moments, likelihood function, etc.). Also, the possible shapes of this pdf are informative on the nature of the related model. On the other side, the corresponding survival function ( $\mathrm{sf}$ ) is given by

$$
S(x ; \alpha, \beta, \xi)=1-F(x ; \alpha, \beta, \xi)=\frac{1+G(x ; \xi)^{\alpha}-2 G(x ; \xi)^{\beta}}{1+G(x ; \xi)^{\alpha}}, \quad x \in \mathbb{R} .
$$

The corresponding hazard rate function (hrf), reverse hazard rate function (rhrf) and cumulative hazard rate function (chrf) are, respectively, given by

$$
\begin{gathered}
h(x ; \alpha, \beta, \xi)=\frac{f(x ; \alpha, \beta, \xi)}{S(x ; \alpha, \beta, \xi)}=\frac{2 G(x ; \xi)^{\beta-1} g(x ; \xi)\left[\beta+(\beta-\alpha) G(x ; \xi)^{\alpha}\right]}{\left[1+G(x ; \xi)^{\alpha}-2 G(x ; \xi)^{\beta}\right]\left[1+G(x ; \xi)^{\alpha}\right]^{2}} \\
r(x ; \alpha, \beta, \xi)=\frac{g(x ; \xi)\left[\beta+(\beta-\alpha) G(x ; \xi)^{\alpha}\right]}{G(x ; \xi)\left[1+G(x ; \xi)^{\alpha}\right]}
\end{gathered}
$$

and

$$
H(x ; \alpha, \beta, \xi)=-\log [S(x ; \alpha, \beta, \xi)]=\log \left[1+G(x ; \xi)^{\alpha}\right]-\log \left[1+G(x ; \xi)^{\alpha}-2 G(x ; \xi)^{\beta}\right], \quad x \in \mathbb{R} .
$$

When the support of the distribution related to $G(x ; \xi)$ is $(0,+\infty)$, as any lifetime baseline distribution, the sf, hrf, rhrf and chrf are involved in a plethora of applications in survival analysis (see [12]).

The quantile function ( $\mathrm{qf}$ ), say $Q(u ; \alpha, \beta, \xi)$, can be obtained by inverting $F(x ; \alpha, \beta, \xi)$, i.e., it satisfies the following non-linear equation: $F(Q(u ; \alpha, \beta, \xi) ; \alpha, \beta, \xi)=u$ for $u \in(0,1)$. In full generality, there is no closed-form for this function. As basic approach, the only identifying closed-form are for $\beta=\alpha$, where $Q(u ; \alpha, \beta, \xi)=Q_{G}\left([u /(2-u)]^{1 / \alpha} ; \xi\right)$, and for $\alpha=2 \beta$, where $Q(u ; \alpha, \beta, \xi)=$ $Q_{G}\left(\left[\left(1-\sqrt{1-u^{2}}\right) / u\right]^{1 / \beta} ; \xi\right)$, and $Q_{G}(u ; \xi)$ denotes the qf corresponding to $G(x ; \xi)$. Thanks to the qf, one can express the quartiles and octiles of the considered distribution, as well as several measures of skewness and asymmetry, such as the Bowley or MacGillivray skewness (see [13]). 


\section{Mathematical Properties}

In this section, we derive the main mathematical properties of the RE-G family, with discussions.

\subsection{Stochastic Ordering Results}

Here, we investigate some stochastic ordering relation between the RE-G and Exp-G families according to the values of $\alpha$ and $\beta$.

First of all, as alpha results,

- $\quad$ if $\alpha \geq 0$, we have $G(x ; \xi)^{\beta} \leq F(x ; \alpha, \beta, \xi)$,

- $\quad$ if $\alpha<0$, the reversed inequality holds: $G(x ; \xi)^{\beta} \geq F(x ; \alpha, \beta, \xi)$.

Indeed, if $\alpha \geq 0$, we have $G(x ; \xi)^{\alpha} \leq 1$ so $1+G(x ; \xi)^{\alpha} \leq 2$ and if $\alpha<0$, we have $G(x ; \xi)^{\alpha} \geq 1$ so $1+G(x ; \xi)^{\alpha} \geq 2$. We thus see the importance of the parameter $\alpha$ according to its sign, regarding an immediate stochastic hierarchy between the RE-G and Exp-G families.

The following result proposes a refinement of the upper bound for $F(x ; \alpha, \beta, \xi)$.

Proposition 1. For any $\alpha \in \mathbb{R}$ (such that $\beta \neq 0$ and $\beta \geq \alpha / 2$, or $\beta=0$ and $\alpha<0$ ), we have

$$
F(x ; \alpha, \beta, \xi) \leq G(x ; \xi)^{\beta-\alpha / 2}
$$

Proof. Let us distinguish the two following cases: $\beta \neq 0$ and $\beta \geq \alpha / 2$ on the one hand, and $\beta=0$ and $\alpha<0$ on the other hand.

Case I: $\beta \neq 0$ and $\beta \geq \alpha / 2$. We can express $F(x ; \alpha, \beta, \xi)$ as

$$
F(x ; \alpha, \beta, \xi)=\frac{2 G(x ; \xi)^{\alpha / 2}}{1+G(x ; \xi)^{\alpha}} G(x ; \xi)^{\beta-\alpha / 2} \text {. }
$$

Now remark that $2 G(x ; \xi)^{\alpha / 2} /\left[1+G(x ; \xi)^{\alpha}\right] \leq 1$ since $\left[1-G(x ; \xi)^{\alpha / 2}\right]^{2} \geq 0$, implying the desired result.

Case II: $\beta=0$ and $\alpha<0$. We can express $F(x ; \alpha, 0, \xi)$ as

$$
F(x ; \alpha, 0, \xi)=\frac{G(x ; \xi)^{-\alpha}}{G(x ; \xi)^{-\alpha}} \times \frac{2}{1+G(x ; \xi)^{\alpha}}=\frac{2 G(x ; \xi)^{-\alpha / 2}}{1+G(x ; \xi)^{-\alpha}} G(x ; \xi)^{-\alpha / 2} .
$$

Since $2 G(x ; \xi)^{-\alpha / 2} /\left[1+G(x ; \xi)^{-\alpha}\right] \leq 1$, the desired result follows.

This ends the proof of Proposition 1.

Hence, Proposition 1 shows the deep relation existing between the RE-G and Exp-G families. Also, for practical purposes, the above result proves that the RE-G family reached different targets in terms of modelling in comparison to the Exp-G family; the RE-G models can be more adequate to the Exp-G models, depending on the nature of the data.

\subsection{On the RE-G pdf}

Let us now present some properties on the curvature of $f(x ; \alpha, \beta, \xi)$, which can be informative for fitting purposes (uni/multimodality nature, polynomial/exponential decay on the tails, etc.).

First of all, when $x \rightarrow-\infty$, let us distinguish the cases $\alpha>0$ on the one hand, then $\alpha=0$ and $\alpha<0$ on the other hand.

- If $\alpha>0$, we have $f(x ; \alpha, \beta, \xi) \sim 2 \beta G(x ; \xi)^{\beta-1} g(x ; \xi)$.

- If $\alpha=0$, we have $f(x ; \alpha, \beta, \xi)=\beta G(x ; \xi)^{\beta-1} g(x ; \xi)$.

- If $\alpha<0$, we have $f(x ; \alpha, \beta, \xi) \sim 2(\beta-\alpha) G(x ; \xi)^{\beta-\alpha-1} g(x ; \xi)$. 
Also, when $x \rightarrow+\infty$, we have $f(x ; \alpha, \beta, \xi) \sim(\beta-\alpha / 2) g(x ; \xi)$. For a given $G(x ; \xi)$, a possible polynomial or exponential decay of the limiting functions characterize the heaviness nature of the tails of the corresponding RE-G distribution.

The mode(s) of a distribution gives an important information of the related model, mainly on its uni/multimodality nature. Here, the mode(s) is(are) given by the critical point(s) of $f(x ; \alpha, \beta, \xi)$, which is (are) given by the solution(s) of the following non-linear equation: $\{\log [f(x ; \alpha, \beta, \xi)]\}^{\prime}=0$, with derivative with respect to $x$, where

$$
\begin{aligned}
\{\log [f(x ; \alpha, \beta, \xi)]\}^{\prime} & =(\beta-1) \frac{g(x ; \xi)}{G(x ; \xi)}+\frac{g^{\prime}(x ; \xi)}{g(x ; \xi)}+(\beta-\alpha) \alpha \frac{g(x ; \xi) G(x ; \xi)^{\alpha-1}}{\beta+(\beta-\alpha) G(x ; \xi)^{\alpha}} \\
& -2 \alpha \frac{g(x ; \xi) G(x ; \xi)^{\alpha-1}}{1+G(x ; \xi)^{\alpha}} .
\end{aligned}
$$

Then, a mode, say $x_{m}$ is a local maximum if $\left.\{\log [f(x ; \alpha, \beta, \xi)]\}^{\prime \prime}\right|_{x=x_{m}}<0$, it is a local minimum if $\left.\{\log [f(x ; \alpha, \beta, \xi)]\}^{\prime \prime}\right|_{x=x_{m}}>0$ and it is a point of inflexion if $\left.\{\log [f(x ; \alpha, \beta, \xi)]\}^{\prime \prime}\right|_{x=x_{m}}=0$. For a given baseline distribution and parameters, the use of a mathematical software is required to determine the numerical value of a mode.

\subsection{On the RE-G hrf}

Now, let us present some properties on the curvature on $h(x ; \alpha, \beta, \xi)$, which is informative on several survival analysis aspects (see [12]). When $x \rightarrow-\infty$, let us distinguish the cases $\alpha>0$ on the one hand, then $\alpha=0$ and $\alpha<0$ on the other hand.

- If $\alpha>0$, we have $h(x ; \alpha, \beta, \xi) \sim 2 \beta G(x ; \xi)^{\beta-1} g(x ; \xi)$.

- If $\alpha=0$, we have $h(x ; \alpha, \beta, \xi)=\beta G(x ; \xi)^{\beta-1} g(x ; \xi)$.

- If $\alpha<0$, we have $h(x ; \alpha, \beta, \xi) \sim 2(\beta-\alpha) G(x ; \xi)^{\beta-\alpha-1} g(x ; \xi)$.

Also, when $x \rightarrow+\infty$, we have $h(x ; \alpha, \beta, \xi) \sim h_{G}(x ; \xi)$, where $h_{G}(x ; \xi)=g(x ; \xi) /[1-G(x ; \xi)]$, i.e., the hrf corresponding to $G(x ; \xi)$.

The critical point(s) of $h(x ; \alpha, \beta, \xi)$ is (are) given by the solution(s) of the following equation: $\{\log [h(x ; \alpha, \beta, \xi)]\}^{\prime}=0$, where

$$
\begin{aligned}
\{\log [h(x ; \alpha, \beta, \xi)]\}^{\prime} & =(\beta-1) \frac{g(x ; \xi)}{G(x ; \xi)}+\frac{g^{\prime}(x ; \xi)}{g(x ; \xi)}+(\beta-\alpha) \alpha \frac{g(x ; \xi) G(x ; \xi)^{\alpha-1}}{\beta+(\beta-\alpha) G(x ; \xi)^{\alpha}} \\
& -2 \alpha \frac{g(x ; \xi) G(x ; \xi)^{\alpha-1}}{1+G(x ; \xi)^{\alpha}}+\frac{2 G(x ; \xi)^{\beta-1} g(x ; \xi)\left[\beta+(\beta-\alpha) G(x ; \xi)^{\alpha}\right]}{\left[1+G(x ; \xi)^{\alpha}-2 G(x ; \xi)^{\beta}\right]\left[1+G(x ; \xi)^{\alpha}\right]}
\end{aligned}
$$

The nature of a critical point, say $x_{c}$, can be determined by studying the sign of $\left.\{\log [h(x ; \alpha, \beta, \xi)]\}^{\prime \prime}\right|_{x=x_{c}}$. Again, there is no closed-form for $x_{c}$; a mathematical software seems necessary to have an efficient numerical approximation.

\subsection{Series Expansions}

Now, we claim that the pdf of the RE-G family can be expressed as an infinite linear combination of pdfs of the Exp-G family, in a similar fashion to the pdfs of the families developed by $[8,14,15]$, among others. This is formulated in the result below.

Proposition 2. There exist two sequences of real numbers $\left(u_{k}\right)_{k \in \mathbb{N}}$ and $\left(v_{k}\right)_{k \in \mathbb{N}}$ such that, for $x$ satisfying $G(x ; \xi) \in(0,1)$ (excluding the limit cases), we have

$$
f(x ; \alpha, \beta, \xi)=\sum_{k=0}^{+\infty} u_{k} \pi_{v_{k}}(x ; \xi),
$$


where $\pi_{\gamma}(x ; \xi)=\gamma g(x ; \xi) G(x ; \xi)^{\gamma-1}$ is the pdf of the Exp-G family with power parameter $\gamma>0$.

Proof. Let us distinguish the cases $\alpha>0$ on the one hand, then $\alpha=0$ and $\alpha<0$ on the other hand.

Case I: $\alpha>0$. Since $G(x ; \xi) \in(0,1)$, the standard power series expansion gives

$$
F(x ; \alpha, \beta, \xi)=2 G(x ; \xi)^{\beta} \sum_{k=0}^{+\infty}(-1)^{k} G(x ; \xi)^{\alpha k}=\sum_{k=0}^{+\infty}\left[2(-1)^{k}\right] G(x ; \xi)^{\alpha k+\beta} .
$$

Upon differentiation with respect to $x$, we get

$$
f(x ; \alpha, \beta, \xi)=\sum_{k=0}^{+\infty}\left[2(-1)^{k}\right](\alpha k+\beta) g(x ; \xi) G(x ; \xi)^{\alpha k+\beta-1} .
$$

Hence, we can take $u_{k}=2(-1)^{k}$ and $v_{k}=\alpha k+\beta$.

Case II: $a=0$. We have immediately $f(x ; 0, \beta, \xi)=\pi_{\beta}(x ; \xi)$, so the desired result holds with $u_{0}=1$ and $u_{k}=0$ for any $k \geq 1$, and $v_{0}=\beta$.

Case III: $a<0$. By using again the standard power series expansion, we get

$$
\begin{aligned}
F(x ; \alpha, \beta, \xi) & =\frac{G(x ; \xi)^{-\alpha}}{G(x ; \xi)^{-\alpha}} \times \frac{2 G(x ; \xi)^{\beta}}{1+G(x ; \xi)^{\alpha}}=\frac{2 G(x ; \xi)^{\beta-\alpha}}{1+G(x ; \xi)^{-\alpha}} \\
& =2 G(x ; \xi)^{\beta-\alpha} \sum_{k=0}^{+\infty}(-1)^{k} G(x ; \xi)^{-\alpha k}=\sum_{k=0}^{+\infty}\left[2(-1)^{k}\right] G(x ; \xi)^{-\alpha(k+1)+\beta} .
\end{aligned}
$$

Upon differentiation with respect to $x$, we get

$$
f(x ; \alpha, \beta, \xi)=\sum_{k=0}^{+\infty}\left[2(-1)^{k}\right][-\alpha(k+1)+\beta] g(x ; \xi) G(x ; \xi)^{-\alpha(k+1)+\beta-1} .
$$

Hence, we can take $u_{k}=2(-1)^{k}$ and $v_{k}=-\alpha(k+1)+\beta$.

This ends the proof of Proposition 2.

The interest of Proposition 2 is the use of some well-known properties and definitions of the Exp-G family to derive those of the RE-G family. This point is illustrated for the moments and some crucial functions in the next subsection.

\subsection{Moments: Related Measures and Functions}

Let $X$ be a random variable having the cdf of the RE-G family, i.e., given by (2). Then, for any function $\phi(x)$, by the transfer theorem, the expectation of $\phi(X)$ is given by

$$
\begin{aligned}
\Theta_{\phi}(X) & =E[\phi(X)]=\int_{-\infty}^{+\infty} \phi(x) f(x ; \alpha, \beta, \xi) d x \\
& =\int_{-\infty}^{+\infty} \phi(x) \frac{2 G(x ; \xi)^{\beta-1} g(x ; \xi)\left[\beta+(\beta-\alpha) G(x ; \xi)^{\alpha}\right]}{\left[1+G(x ; \xi)^{\alpha}\right]^{2}} d x,
\end{aligned}
$$

(provided that it exists). The considered domain of integration is $\mathbb{R}$ in full generally; it can be reduced, depending on the supports of $\phi(x)$ and $g(x ; \xi)$ only. By using the change of variables $u=G(x ; \xi)$, it can also be expressed as

$$
\Theta_{\phi}(X)=\int_{0}^{1} \phi\left[Q_{G}(u ; \xi)\right] \frac{2 u^{\beta-1}\left[\beta+(\beta-\alpha) u^{\alpha}\right]}{\left(1+u^{\alpha}\right)^{2}} d u .
$$


For given $G(x ; \xi)$ and parameters, we can provide a numerical evaluation of this integral by using any mathematical software.

For analytical purposes, Proposition 2 can be of interest; it implies that

$$
\Theta_{\phi}(X)=\sum_{k=0}^{+\infty} u_{k} \int_{-\infty}^{+\infty} \phi(x) \pi_{v_{k}}(x ; \xi) d x \approx \sum_{k=0}^{K} u_{k} \int_{-\infty}^{+\infty} \phi(x) \pi_{v_{k}}(x ; \xi) d x
$$

for a large enough integer $K$. From the numerical point of view, this approximation may be more efficient that compute directly the integral form of $\Theta_{\phi}(X)$, which can be prone to rounding off errors, as discussed in [14]. Some notable measures and functions derived to $\Theta_{\phi}(X)$ are listed below. By taking $\phi(x)=x, \Theta_{\phi}(X)$ becomes the mean of $X$ denoted by $\mu_{*}$, by choosing $\phi(x)=x^{m}$, we obtain the $m^{\text {th }}$ raw moment of $X$, by taking $\phi(x)=\left(x-\mu_{*}\right)^{m}, \Theta_{\phi}(X)$ becomes the $m^{\text {th }}$ central moment of $X$, the $m^{\text {th }}$ incomplete moment with respect to $t$ follows by taking $\phi(x)=x^{m}$ if $x \leq t$, and 0 elsewhere, and, by choosing $\phi(x)=e^{i t x}, i=\sqrt{-1}$, we get the characteristic function of $X$ with respect to $t$. Further applications of these measures and functions under the forms (4) can be found in $[8,14,15]$, among others.

\subsection{Maximum Likelihood Method}

Here, we adopt a general statistical point view. We consider the RE-G models and investigate the estimation of the models parameters by a very efficient estimation method: the maximum likelihood method, for complete sample only. Let $x_{1}, \ldots, x_{n}$ be $n$ independent observations of a random variable having the pdf of the RE-G family, i.e., given by (3). Then, the log-likelihood function is defined by

$$
\begin{aligned}
\ell(\alpha, \beta, \xi) & =\sum_{i=1}^{n} \log \left[f\left(x_{i} ; \alpha, \beta, \xi\right)\right]=n \log (2)+(\beta-1) \sum_{i=1}^{n} \log \left[G\left(x_{i} ; \xi\right)\right]+\sum_{i=1}^{n} \log \left[g\left(x_{i} ; \xi\right)\right] \\
& +\sum_{i=1}^{n} \log \left[\beta+(\beta-\alpha) G\left(x_{i} ; \xi\right)^{\alpha}\right]-2 \sum_{i=1}^{n} \log \left[1+G\left(x_{i} ; \xi\right)^{\alpha}\right] .
\end{aligned}
$$

Hence, the maximum likelihood estimates (MLEs) of $\alpha, \beta$ and $\xi$, say $\hat{\alpha}, \hat{\beta}$ and $\hat{\xi}$, respectively, are defined by $(\hat{\alpha}, \hat{\beta}, \hat{\xi})=\operatorname{argmax}_{\alpha, \beta, \xi} \ell(\alpha, \beta, \xi)$. This maximization can be performed either directly by using any statistical software such as $\mathrm{R}$ (with the package AdequacyModel) or SAS (with the procedure PROC NLMIXED), or by solving the nonlinear likelihood equations obtained by differentiating $\ell(\alpha, \beta, \xi)$ with respect to the model parameters. In this regard, the score function is useful; it can be expressed as $U(\alpha, \beta, \xi)=(\partial \ell(\alpha, \beta, \xi) / \partial \alpha, \partial \ell(\alpha, \beta, \xi) / \partial \beta, \partial \ell(\alpha, \beta, \xi) / \partial \xi)$, whose elements are given in Appendix A. Thus, the solutions of the system of non-linear equations: $U(\alpha, \beta, \xi)=\overrightarrow{0}$ with respect to $\alpha, \beta$ and $\xi$ gives $\hat{\alpha}, \hat{\beta}$ and $\hat{\xi}$. Also, the observed information matrix can be expressed analytically, allowing to define the corresponding standard errors, and so on. The complete theory can be found in [16].

\section{Numerical Studies}

This section is devoted to the applicability of the RE-G family in a concrete statistical setting. First of all, we introduce a special member of interest, then we investigate the efficiency of the MLEs of the related parameters and analyze three practical data sets, with discussions.

\subsection{The REW Distribution}

For practical purposes, we pay a particular attention on special member of the RE-G family defined with the Weibull distribution with parameters $\mu>0$ and $\theta>0$, as baseline. We call it the REW distribution. Thus, we aim to improve some characteristics of the Weibull distribution (and the related 
model as well), such as the skewness, kurtosis and heaviness of the tails, by tuning the parameters $\alpha$ and $\beta$ in (2). Hence, by using (2), the REW distribution is defined by the following cdf and pdf:

$$
F(x ; \alpha, \beta, \mu, \theta)=\frac{2 G(x ; \mu, \theta)^{\beta}}{1+G(x ; \mu, \theta)^{\alpha}}, \quad x>0
$$

and

$$
f(x ; \alpha, \beta, \mu, \theta)=\frac{2 G(x ; \mu, \theta)^{\beta-1} g(x ; \mu, \theta)\left[\beta+(\beta-\alpha) G(x ; \mu, \theta)^{\alpha}\right]}{\left[1+G(x ; \mu, \theta)^{\alpha}\right]^{2}},
$$

respectively, where

$$
G(x ; \mu, \theta)=1-e^{-\mu x^{\theta}}, \quad g(x ; \mu, \theta)=\mu \theta x^{\theta-1} e^{-\mu x^{\theta}}, \quad x>0 .
$$

Here, $\mu>0$ is a scale parameter, and $\beta, \theta>0$ and $\alpha \in \mathbb{R}$ with $\beta \geq \alpha / 2$ are shape parameters $(\alpha$ can be negative). Also, the corresponding hrf is given by

$$
h(x ; \alpha, \beta, \mu, \theta)=\frac{2 G(x ; \mu, \theta)^{\beta-1} g(x ; \mu, \theta)\left[\beta+(\beta-\alpha) G(x ; \mu, \theta)^{\alpha}\right]}{\left[1+G(x ; \mu, \theta)^{\alpha}-2 G(x ; \mu, \theta)^{\beta}\right]\left[1+G(x ; \mu, \theta)^{\alpha}\right]} .
$$

The REW distribution constitutes a new four-parameters lifetime distribution, whose pdf and hrf enjoy attractive flexible properties for modelling purposes, as illustrated in Figures 1 and 2.
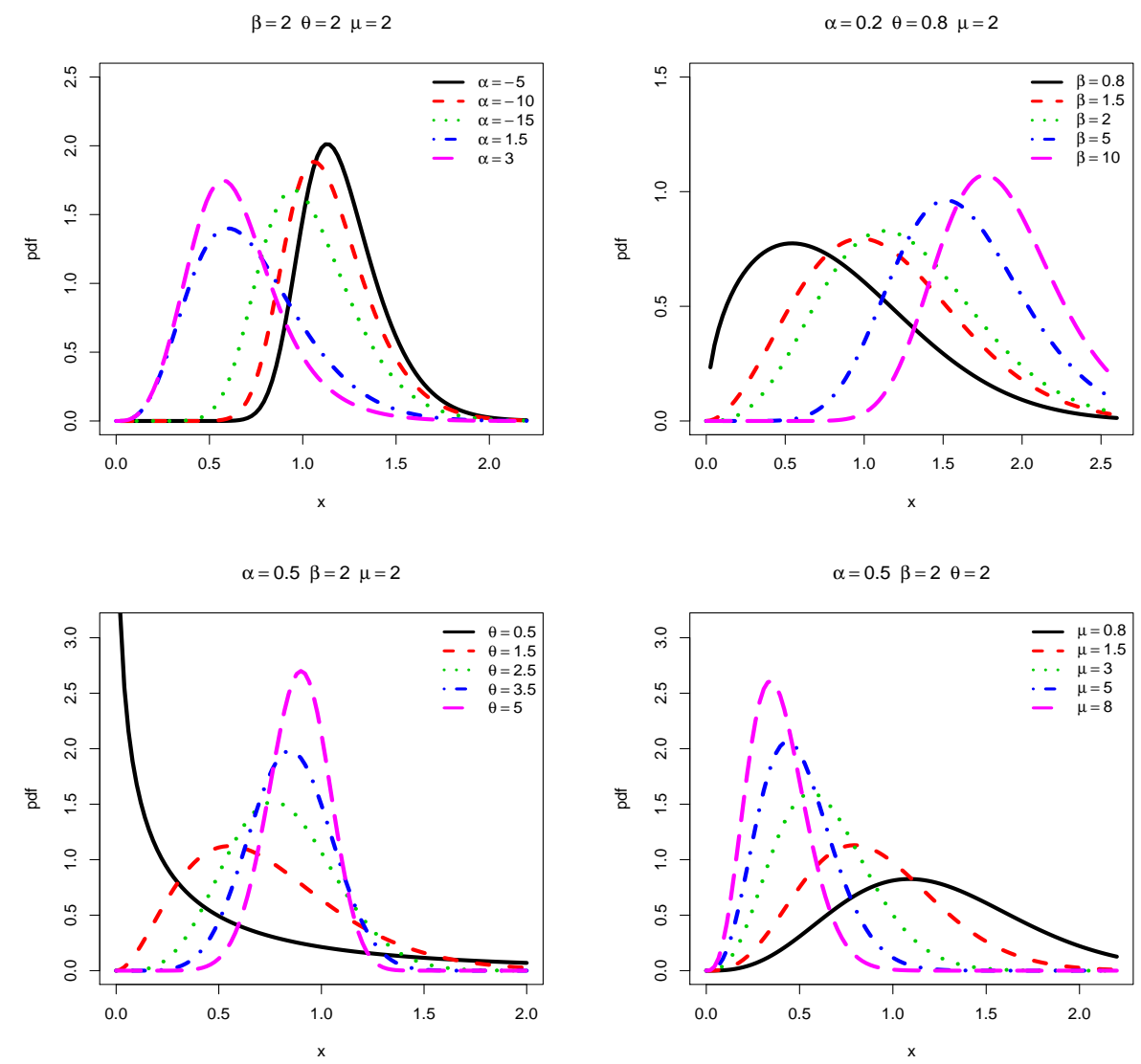

Figure 1. Plots of the pdf of the REW distribution for some values of the parameters. 

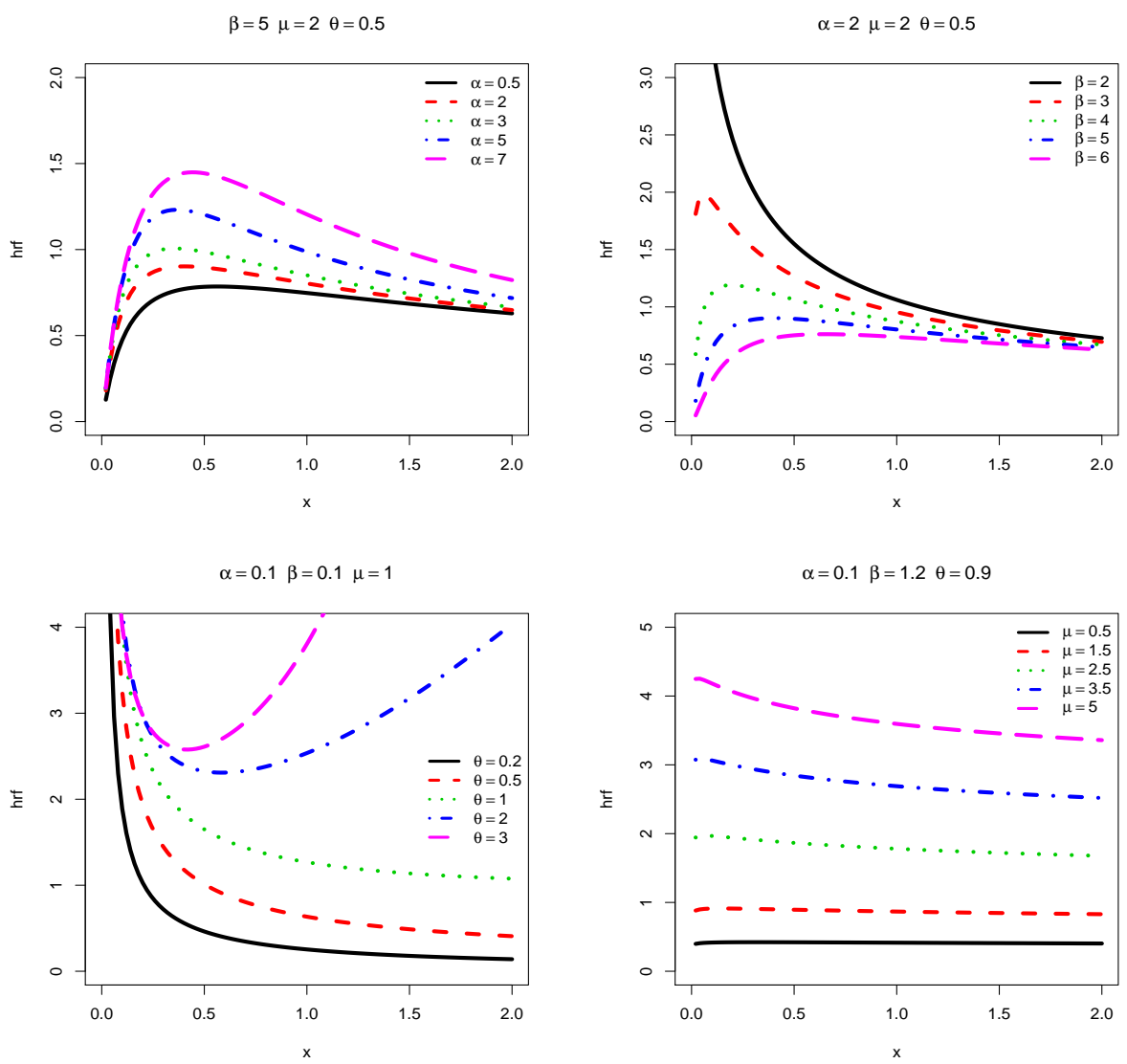

Figure 2. Plots of the hrf of the REW distribution for some values of the parameters.

From Figure 1, we see that the pdf be can left skewed, right skewed, near symmetrical and also presents reverse J shapes. The tails of the distribution can be more or less heavy, mainly depending on the values of $\mu$; the tails is heavier as $\mu$ decreases. We also observe various degrees of leptokurtic, mesokurtic and platykurtic shapes. As a notable fact, Figure 2 reveals that the REW distribution has all the possible monotonic and non-monotonic hazard rate shapes, such as increasing, decreasing, decreasing-increasing-decreasing, constant, bathtub and upside-down bathtub shapes.

In addition, all the general theoretical properties presented in the above section can be applied without efforts.

Naturally, one can consider other special members of the RE-G family by choosing other baseline distributions, depending on the context of the study. In particular, the conjoint actions of the parameters $\alpha$ and $\beta$ can be of interest to modulate the properties of the tails of distributions somehow rigid on this aspect. We may think to the ("only" right heavy-tailed) Pareto distribution, whose extensions has been the object of all the attentions these last years. We may refer to $[9,17]$, and the references therein.

The next of the study focus on the numerical aspects of the REW model. The software R is used (see [18]), with the package AdequacyModel, allowing to analyze the adequacy of statistical models via several statistics for a given data set. For all the technical details, we refer the reader to [19].

\subsection{Simulation Study}

Here, turning out the REW distribution as a statistical model with parameters $\alpha, \beta, \mu$ and $\theta$, we propose a Monte-Carlo simulation study to evaluate the accuracy of the MLEs $\hat{\alpha}, \hat{\beta}, \hat{\mu}$ and $\hat{\theta}$, as described in Section 3.6. Under some technical conditions, it is well-known that the MLEs are asymptotically unbiased and convergent. We illustrate these properties by proceeding as follows. For $n \in\{10,20,30,50,100,200,300,500,1000,2000,3000,5000\}$, we generate $N=10,000$ samples of independent values of size $n$ from the REW distribution for the following eight sets of parameters: 
- $\operatorname{Set} 1(\alpha=1.2, \beta=0.8, \mu=0.5, \theta=0.5), \operatorname{Set} 2(\alpha=1.5, \beta=1.5, \mu=0.5, \theta=0.5)$,

- $\operatorname{Set} 3(\alpha=2.0, \beta=1.5, \mu=0.5, \theta=0.5), \operatorname{Set} 4(\alpha=1.2, \beta=1.5, \mu=0.5, \theta=0.5)$,

- $\operatorname{Set} 5(\alpha=1.2, \beta=0.8, \mu=0.8, \theta=0.8), \operatorname{Set} 6(\alpha=1.5, \beta=1.5, \mu=0.8, \theta=0.8)$,

- $\operatorname{Set7}(\alpha=0.5, \beta=1.5, \mu=0.8, \theta=0.8), \operatorname{Set} 8(\alpha=1.2, \beta=1.5, \mu=0.8, \theta=0.8)$.

Then, the (average of the) MLEs and the corresponding mean squared errors (MSEs) are determined for each sample. The numerical results are collected in Tables 1 and 2.

Table 1. Simulations for the REW model: maximum likelihood estimates (MLEs) and mean squared errors (MSEs) with the sets of parameters Set1, Set2, Set3 and Set4.

\begin{tabular}{|c|c|c|c|c|c|c|c|c|}
\hline \multirow{2}{*}{$n$} & \multicolumn{2}{|c|}{ Set1 } & \multicolumn{2}{|c|}{ Set2 } & \multicolumn{2}{|c|}{ Set3 } & \multicolumn{2}{|c|}{ Set4 } \\
\hline & MLE & MSE & MLE & MSE & MLE & MSE & MLE & MSE \\
\hline \multirow{4}{*}{10} & 1.31976 & 0.20969 & 1.65718 & 0.32829 & 2.23432 & 0.95120 & 1.34140 & 0.17560 \\
\hline & 0.92462 & 0.19557 & 1.65988 & 1.06191 & 1.91209 & 10.15240 & 1.61679 & 0.25485 \\
\hline & 0.63744 & 0.14013 & 0.57193 & 0.09347 & 0.57197 & 0.08969 & 0.62512 & 0.10805 \\
\hline & 0.54963 & 0.03722 & 0.48575 & 0.01688 & 0.48812 & 0.03115 & 0.51703 & 0.01372 \\
\hline \multirow{4}{*}{20} & 1.26755 & 0.08585 & 1.55946 & 0.09287 & 2.05720 & 0.19105 & 1.27318 & 0.13865 \\
\hline & 0.84839 & 0.05330 & 1.59549 & 0.20253 & 1.72352 & 0.62737 & 1.62612 & 0.24885 \\
\hline & 0.56193 & 0.04765 & 0.55215 & 0.03640 & 0.52509 & 0.02135 & 0.52403 & 0.01894 \\
\hline & 0.51070 & 0.01828 & 0.48932 & 0.01080 & 0.46634 & 0.01543 & 0.50848 & 0.01087 \\
\hline \multirow{4}{*}{30} & 1.24023 & 0.05841 & 1.51725 & 0.09215 & 2.12476 & 0.22875 & 1.24851 & 0.05840 \\
\hline & 0.83430 & 0.03799 & 1.61337 & 0.13463 & 1.56983 & 0.18756 & 1.55033 & 0.10354 \\
\hline & 0.53329 & 0.02514 & 0.51904 & 0.01608 & 0.54111 & 0.02392 & 0.53468 & 0.01995 \\
\hline & 0.50043 & 0.01416 & 0.49145 & 0.00648 & 0.45122 & 0.00938 & 0.49928 & 0.00667 \\
\hline \multirow{4}{*}{50} & 1.22022 & 0.03004 & 1.50542 & 0.06575 & 2.06140 & 0.09455 & 1.22643 & 0.03254 \\
\hline & 0.82281 & 0.01649 & 1.56373 & 0.08696 & 1.55281 & 0.10487 & 1.53129 & 0.05152 \\
\hline & 0.52581 & 0.01425 & 0.50473 & 0.01137 & 0.53381 & 0.01662 & 0.51614 & 0.00747 \\
\hline & 0.49444 & 0.00777 & 0.47998 & 0.00461 & 0.44677 & 0.00699 & 0.49706 & 0.00288 \\
\hline \multirow{4}{*}{100} & 1.21332 & 0.01523 & 1.51241 & 0.03139 & 2.03416 & 0.04672 & 1.19958 & 0.01171 \\
\hline & 0.80755 & 0.00836 & 1.52931 & 0.03405 & 1.50795 & 0.04534 & 1.53265 & 0.02624 \\
\hline & 0.50853 & 0.00562 & 0.50948 & 0.00593 & 0.50600 & 0.00420 & 0.50613 & 0.00355 \\
\hline & 0.48272 & 0.00414 & 0.47693 & 0.00295 & 0.43756 & 0.00591 & 0.49738 & 0.00183 \\
\hline \multirow{4}{*}{200} & 1.20337 & 0.00701 & 1.49122 & 0.01196 & 2.01550 & 0.01845 & 1.19102 & 0.00670 \\
\hline & 0.80374 & 0.00359 & 1.50672 & 0.01441 & 1.50491 & 0.01804 & 1.54106 & 0.01498 \\
\hline & 0.50365 & 0.00288 & 0.49548 & 0.00243 & 0.50354 & 0.00206 & 0.50365 & 0.00230 \\
\hline & 0.47990 & 0.00214 & 0.47106 & 0.00182 & 0.43745 & 0.00480 & 0.49773 & 0.00113 \\
\hline \multirow{4}{*}{300} & 1.20450 & 0.00482 & 1.51361 & 0.00626 & 1.98904 & 0.01367 & 1.21308 & 0.00441 \\
\hline & 0.80290 & 0.00238 & 1.51080 & 0.00981 & 1.52714 & 0.01683 & 1.50609 & 0.00814 \\
\hline & 0.50443 & 0.00190 & 0.51012 & 0.00158 & 0.49999 & 0.00137 & 0.50914 & 0.00152 \\
\hline & 0.47982 & 0.00158 & 0.47381 & 0.00140 & 0.44155 & 0.00424 & 0.49111 & 0.00072 \\
\hline \multirow{4}{*}{500} & 1.20005 & 0.00289 & 1.51469 & 0.00497 & 2.00726 & 0.00719 & 1.19714 & 0.00256 \\
\hline & 0.80281 & 0.00150 & 1.49185 & 0.00604 & 1.50562 & 0.00818 & 1.50801 & 0.00504 \\
\hline & 0.50172 & 0.00107 & 0.50402 & 0.00075 & 0.50364 & 0.00096 & 0.49962 & 0.00078 \\
\hline & 0.47900 & 0.00117 & 0.46906 & 0.00136 & 0.43844 & 0.00424 & 0.49047 & 0.00045 \\
\hline
\end{tabular}


Table 1. Cont

\begin{tabular}{ccccccccc}
\hline \multirow{2}{*}{$n$} & \multicolumn{2}{c}{ Set1 } & \multicolumn{2}{c}{ Set2 } & \multicolumn{2}{c}{ Set3 } & \multicolumn{2}{c}{ Set4 } \\
\cline { 2 - 9 } & MLE & MSE & MLE & MSE & MLE & MSE & MLE & MSE \\
\hline \multirow{3}{*}{1000} & 1.20090 & 0.00141 & 1.49981 & 0.00188 & 1.99554 & 0.00362 & 1.20356 & 0.00123 \\
\cline { 2 - 9 } & 0.80096 & 0.00076 & 1.50198 & 0.00268 & 1.50957 & 0.00302 & 1.49484 & 0.00261 \\
\cline { 2 - 9 } & 0.50130 & 0.00054 & 0.50005 & 0.00038 & 0.50127 & 0.00052 & 0.49958 & 0.00031 \\
\cline { 2 - 9 } 2000 & 0.47803 & 0.00086 & 0.47091 & 0.00104 & 0.43919 & 0.00387 & 0.48721 & 0.00036 \\
\hline \multirow{3}{*}{3000} & 1.20076 & 0.00073 & 1.50213 & 0.00102 & 2.00496 & 0.00199 & 1.20334 & 0.00063 \\
\cline { 2 - 9 } & 0.79970 & 0.00036 & 1.49702 & 0.00147 & 1.49945 & 0.00186 & 1.50079 & 0.00108 \\
\cline { 2 - 9 } & 0.49972 & 0.00026 & 0.49950 & 0.00018 & 0.50153 & 0.00021 & 0.50361 & 0.00026 \\
\hline & 0.47654 & 0.00073 & 0.46930 & 0.00104 & 0.43719 & 0.00403 & 0.49053 & 0.00019 \\
\cline { 2 - 9 } & 0.79948 & 0.00026 & 1.51017 & 0.00095 & 1.50398 & 0.00143 & 1.50236 & 0.00068 \\
\hline & 0.49994 & 0.00018 & 0.49841 & 0.00018 & 0.50434 & 0.00018 & 0.50013 & 0.00014 \\
\hline \multirow{3}{*}{5000} & 0.47650 & 0.00068 & 0.47211 & 0.00084 & 0.43901 & 0.00380 & 0.48988 & 0.00016 \\
\cline { 2 - 9 } & 1.20050 & 0.00029 & 1.49910 & 0.00044 & 1.99529 & 0.00083 & 1.20051 & 0.00035 \\
\hline & 0.79970 & 0.00014 & 1.50123 & 0.00057 & 1.50363 & 0.00079 & 1.49956 & 0.00064 \\
\hline & 0.49976 & 0.00011 & 0.49981 & 0.00009 & 0.49894 & 0.00010 & 0.50055 & 0.00010 \\
\hline & 0.47649 & 0.00062 & 0.47044 & 0.00091 & 0.43776 & 0.00391 & 0.48913 & 0.00016 \\
\hline
\end{tabular}

Table 2. Simulations for the REW model: MLEs and MSEs with the sets of parameters Set5, Set6, Set7 and Set8.

\begin{tabular}{|c|c|c|c|c|c|c|c|c|}
\hline \multirow{2}{*}{$n$} & \multicolumn{2}{|c|}{ Set5 } & \multicolumn{2}{|c|}{ Set6 } & \multicolumn{2}{|c|}{ Set7 } & \multicolumn{2}{|c|}{ Set8 } \\
\hline & MLE & MSE & MLE & MSE & MLE & MSE & MLE & MSE \\
\hline \multirow{4}{*}{10} & 1.32799 & 0.32521 & 1.63504 & 0.25842 & 0.57191 & 0.04304 & 1.32992 & 0.20068 \\
\hline & 0.97519 & 0.17319 & 1.78943 & 0.79665 & 1.56281 & 0.13696 & 1.60799 & 0.27552 \\
\hline & 1.09822 & 0.41755 & 0.95780 & 0.18136 & 0.95338 & 0.24007 & 0.91000 & 0.16596 \\
\hline & 1.03531 & 0.32488 & 0.86786 & 0.16398 & 0.90247 & 0.05940 & 0.83769 & 0.09756 \\
\hline \multirow{4}{*}{20} & 1.27713 & 0.09060 & 1.58165 & 0.13866 & 0.54253 & 0.02022 & 1.24066 & 0.08531 \\
\hline & 0.82879 & 0.04342 & 1.64576 & 0.39060 & 1.51620 & 0.06415 & 1.61416 & 0.22844 \\
\hline & 0.88404 & 0.11136 & 0.85742 & 0.05858 & 0.87833 & 0.07836 & 0.82854 & 0.04724 \\
\hline & 0.80472 & 0.07089 & 0.79330 & 0.08132 & 0.87367 & 0.03068 & 0.83567 & 0.06829 \\
\hline \multirow{4}{*}{30} & 1.23593 & 0.05205 & 1.55980 & 0.09931 & 0.51027 & 0.00864 & 1.24367 & 0.05174 \\
\hline & 0.86082 & 0.04045 & 1.61726 & 0.17093 & 1.54061 & 0.04091 & 1.52560 & 0.07546 \\
\hline & 0.89929 & 0.07254 & 0.87512 & 0.04950 & 0.83780 & 0.03727 & 0.82727 & 0.03402 \\
\hline & 0.84757 & 0.07397 & 0.78596 & 0.04175 & 0.89080 & 0.02522 & 0.78334 & 0.02551 \\
\hline \multirow{4}{*}{50} & 1.21763 & 0.03158 & 1.55526 & 0.03914 & 0.50918 & 0.00538 & 1.25554 & 0.03658 \\
\hline & 0.84312 & 0.02099 & 1.51119 & 0.05834 & 1.53389 & 0.02663 & 1.52511 & 0.06835 \\
\hline & 0.87329 & 0.05220 & 0.84497 & 0.02718 & 0.83058 & 0.02694 & 0.85963 & 0.03848 \\
\hline & 0.81029 & 0.03355 & 0.72850 & 0.02282 & 0.88257 & 0.01836 & 0.79156 & 0.02633 \\
\hline
\end{tabular}


Table 2. Cont

\begin{tabular}{|c|c|c|c|c|c|c|c|c|}
\hline \multirow{2}{*}{$n$} & \multicolumn{2}{|c|}{ Set5 } & \multicolumn{2}{|c|}{ Set6 } & \multicolumn{2}{|c|}{ Set7 } & \multicolumn{2}{|c|}{ Set8 } \\
\hline & MLE & MSE & MLE & MSE & MLE & MSE & MLE & MSE \\
\hline \multirow{4}{*}{100} & 1.20294 & 0.01690 & 1.54232 & 0.02343 & 0.50832 & 0.00251 & 1.20689 & 0.01301 \\
\hline & 0.81507 & 0.00797 & 1.50369 & 0.03313 & 1.51037 & 0.00998 & 1.52588 & 0.02450 \\
\hline & 0.81520 & 0.01609 & 0.82906 & 0.01479 & 0.82219 & 0.01153 & 0.81204 & 0.00941 \\
\hline & 0.76410 & 0.01483 & 0.72035 & 0.01577 & 0.86831 & 0.01048 & 0.78314 & 0.00832 \\
\hline \multirow{4}{*}{200} & 1.20821 & 0.00992 & 1.49935 & 0.01498 & 0.49780 & 0.00143 & 1.20074 & 0.00775 \\
\hline & 0.80202 & 0.00446 & 1.52305 & 0.01821 & 1.50955 & 0.00495 & 1.52901 & 0.01610 \\
\hline & 0.80345 & 0.00754 & 0.80269 & 0.00524 & 0.79708 & 0.00599 & 0.81329 & 0.00530 \\
\hline & 0.74732 & 0.00967 & 0.72646 & 0.01003 & 0.86266 & 0.00658 & 0.78747 & 0.00578 \\
\hline \multirow{4}{*}{300} & 1.21978 & 0.00630 & 1.50992 & 0.00885 & 0.50333 & 0.00084 & 1.20019 & 0.00539 \\
\hline & 0.79484 & 0.00263 & 1.50713 & 0.01286 & 1.49149 & 0.00340 & 1.49859 & 0.01029 \\
\hline & 0.80676 & 0.00426 & 0.80646 & 0.00375 & 0.80083 & 0.00290 & 0.79623 & 0.00354 \\
\hline & 0.73960 & 0.00779 & 0.71880 & 0.00999 & 0.85144 & 0.00447 & 0.76624 & 0.00507 \\
\hline \multirow{4}{*}{500} & 1.20444 & 0.00249 & 1.50705 & 0.00421 & 0.49999 & 0.00053 & 1.20514 & 0.00302 \\
\hline & 0.80526 & 0.00131 & 1.50074 & 0.00615 & 1.50305 & 0.00198 & 1.49962 & 0.00530 \\
\hline & 0.81497 & 0.00310 & 0.80406 & 0.00223 & 0.79894 & 0.00199 & 0.80219 & 0.00212 \\
\hline & 0.75415 & 0.00451 & 0.71612 & 0.00877 & 0.85854 & 0.00449 & 0.76656 & 0.00311 \\
\hline \multirow{4}{*}{1000} & 1.19845 & 0.00101 & 1.50271 & 0.00272 & 0.50248 & 0.00022 & 1.20022 & 0.00114 \\
\hline & 0.80184 & 0.00079 & 1.48830 & 0.00315 & 1.49540 & 0.00111 & 1.50216 & 0.00201 \\
\hline & 0.80173 & 0.00183 & 0.79462 & 0.00110 & 0.80180 & 0.00078 & 0.80223 & 0.00129 \\
\hline & 0.74604 & 0.00440 & 0.70872 & 0.00913 & 0.85421 & 0.00351 & 0.76871 & 0.00179 \\
\hline \multirow{4}{*}{2000} & 1.19766 & 0.00087 & 1.49895 & 0.00124 & 0.50115 & 0.00012 & 1.20193 & 0.00075 \\
\hline & 0.80187 & 0.00043 & 1.50566 & 0.00242 & 1.49993 & 0.00051 & 1.50360 & 0.00133 \\
\hline & 0.79947 & 0.00082 & 0.79907 & 0.00042 & 0.80200 & 0.00044 & 0.80418 & 0.00077 \\
\hline & 0.74478 & 0.00378 & 0.71715 & 0.00750 & 0.85781 & 0.00361 & 0.76945 & 0.00146 \\
\hline \multirow{4}{*}{3000} & 1.19673 & 0.00045 & 1.49807 & 0.00063 & 0.50009 & 0.00008 & 1.20220 & 0.00043 \\
\hline & 0.80166 & 0.00027 & 1.50105 & 0.00111 & 1.50210 & 0.00037 & 1.49919 & 0.00086 \\
\hline & 0.79823 & 0.00050 & 0.79871 & 0.00042 & 0.80060 & 0.00031 & 0.80171 & 0.00027 \\
\hline & 0.74406 & 0.00361 & 0.71585 & 0.00741 & 0.85902 & 0.00369 & 0.76656 & 0.00141 \\
\hline \multirow{4}{*}{5000} & 1.20183 & 0.00023 & 1.50050 & 0.00044 & 0.49989 & 0.00004 & 1.20061 & 0.00027 \\
\hline & 0.79836 & 0.00011 & 1.50235 & 0.00062 & 1.49933 & 0.00022 & 1.50039 & 0.00057 \\
\hline & 0.79953 & 0.00024 & 0.80098 & 0.00017 & 0.79959 & 0.00018 & 0.80100 & 0.00021 \\
\hline & 0.74125 & 0.00365 & 0.71655 & 0.00713 & 0.85743 & 0.00341 & 0.76715 & 0.00130 \\
\hline
\end{tabular}

Tables 1 and 2 show that, as the sample size $n$ increases, the MLEs are closed to the true values of the parameters and the corresponding MSEs tend to 0 , which is in concordance with the theoretical properties of the MLEs.

\subsection{Data Fitting}

Application of the REW model is provided by the means of the following three practical data sets.

Data set 1: Carbon dioxide. The first data set represents the annual mean growth rate of carbon dioxide at Mauna Loa (Hawaii). The measurement are given in parts per million year. The data 
are available at the earth system research laboratory website, from the following electronic link: https://www.esrl.noaa.gov/gmd/ccgg/trends/gr.html We have analyzed these data from the period of 1959 to 2014 .

Data set 2: Annual flood discharges. The second data set was originally reported by [20]. It represents the maximum annual flood discharges of the North Saskachevan of the North Saskachevan River at Edmonton, over a period of 47 years. The measurement are given in 1000 cubic feet per second.

Data set 3: Rainfall. The third data consists of the mean of maximum daily rainfall for 30 years (1975-2004) at 35 stations in the middle and west of peninsular Malaysia. This data was recently studied by [21]. The data are given as follows: 1.134, 1.196, 1.181, 1.178, 1.048, 1.077, 0.835, 1.163, $0.880,1.056,1.164,0.914,1.141,1.068,1.007,1.027,1.298,0.842,0.991,0.955,0.703,0.953,1.018$, $1.003,1.106,1.110,1.249,1.092,1.187,1.047,0.989,0.955,1.234,0.937,0.933$.

As immediate remarks, the overall histogram shape of Carbon dioxide is near symmetrical, the one of Annual flood discharges is highly right skewed and the one of Rainfall is moderately left skewed; these data sets are of different nature. In the next, as expected, we show that REW model has the ability to fit these data in an efficiency manner, as developed below.

In this regard, we aim to compare the fits of the REW model with those of five other solid models, also defined with the Weibull distribution as baseline, namely: transmuted-Weibull (TW) model studied by [22], Marshall-Olkin exponential Weibull (MOEW) model introduced by [23], odd log-logistic modified-Weibull (OLLMW) model proposed by [24], Kumaraswamy-Weibull (KW) model introduced by [15] and Beta-Weibull (BW) model proposed by [25].

The values of the MLEs of the models parameters are given (with four decimals) in Tables 3-5 for Carbon dioxide, Annual flood discharges and Rainfall, respectively.

For fitting comparison purposes, we consider the following criteria: complete minus log-likelihood $(-\hat{\ell})$, i.e., the minus log-likelihood of the model taken at the corresponding MLEs, Akaike information criterion (AIC), Cramér-von-Mises (W), Anderson-Darling (AD) and Kolmogorov-Smirnov (KS) statistics, as well as the $p$-value of the related KS test. They are, respectively, defined by

$$
\begin{aligned}
\mathrm{AIC} & =-2 \hat{\ell}+2 p \\
\mathrm{~W} & =\left(\frac{0.5}{n}+1\right)\left[\sum_{i=1}^{n}\left(y_{i}-\frac{2 i-1}{2 n}\right)^{2}+\frac{1}{12 n}\right], \\
\mathrm{AD} & =-\left(\frac{2.25}{n^{2}}+\frac{0.75}{n}+1\right)\left[n+\frac{1}{n} \sum_{i=1}^{n}(2 i-1)\left\{\log \left(y_{i}\right)+\log \left(1-y_{n-i+1}\right)\right\}\right], \\
\mathrm{KS} & =\max \left[\frac{i}{n}-y_{i}, y_{i}-\frac{i-1}{n}\right]
\end{aligned}
$$

and $p$-value $=P\left(D_{n} \geq K S\right)$, with $D_{n}=\sup _{x \in \mathbb{R}}\left|F_{n}(x)-\hat{F}(x)\right|$, where $p$ is the number of parameters of the considered model, $x_{(1)}, \ldots, x_{(n)}$ are the ordered observations, $y_{i}=\hat{F}\left(x_{(i)}\right)$, where $\hat{F}(x)$ denotes the corresponding cdf of the model defined with the corresponding MLEs for the parameters and $F_{n}(x)$ denotes the random empirical cdf. Details on these measures can be found in [26]. We also refer to [27] concerning the KS test. For a given data set and model, the values of these measures are listed in the output of the command goodness.fit of the R package AdequacyModel. 
Table 3. Values of the MLEs of the models parameters and their corresponding SEs (in parentheses) for Carbon dioxide.

\begin{tabular}{ccccccc}
\hline Model & $\boldsymbol{\alpha}$ & $\boldsymbol{\beta}$ & $\boldsymbol{\mu}$ & $\boldsymbol{\theta}$ & $\lambda$ & $\gamma$ \\
\hline REW & 0.5075 & 0.5753 & 0.0288 & 4.5556 & - & - \\
& $(0.3383)$ & $(0.4409)$ & $(0.0857)$ & $(2.6780)$ & - & - \\
TW & 2.7289 & 1.7112 & - & - & 0.0649 & - \\
& $(0.4063)$ & $(0.2223)$ & - & - & $(0.5644)$ & - \\
MOEW & - & - & 1.1019 & 3.3684 & 0.5676 & 325.5881 \\
& - & - & $(0.1932)$ & $(0.8510)$ & $(2.8181)$ & $(25.3028)$ \\
OLLMW & 0.4618 & 0.0061 & 4.3488 & 2.2007 & - & - \\
& $(0.0432)$ & $(0.0063)$ & $(1.2763)$ & $(0.3718)$ & - & - \\
KW & 0.9535 & 0.1730 & 1.3487 & 2.7752 & - & - \\
& $(0.3439)$ & $(0.0546)$ & $(0.1354)$ & $(0.2147)$ & - & - \\
BW & 0.8181 & 0.7930 & 0.2154 & 3.0335 & - & - \\
& $(0.5851)$ & $(3.2477)$ & $(1.0569)$ & $(1.3964)$ & - & - \\
\hline
\end{tabular}

Table 4. Values of the MLEs of the models parameters and their corresponding SEs (in parentheses) for Annual flood discharges.

\begin{tabular}{ccccccc}
\hline Model & $\boldsymbol{\alpha}$ & $\boldsymbol{\beta}$ & $\boldsymbol{\mu}$ & $\boldsymbol{\theta}$ & $\boldsymbol{\lambda}$ & $\gamma$ \\
\hline REW & 93.8263 & 110.4179 & 0.8449 & 0.4564 & - & - \\
& $(3.9839)$ & $(1.1418)$ & $(0.7181)$ & $(0.1409)$ & - & - \\
TW & 1.9474 & 72.9570 & - & - & 0.7044 & - \\
& $(0.1943)$ & $(8.3880)$ & - & - & $(0.2478)$ & - \\
MOEW & - & - & 0.2134 & 0.6563 & 0.8308 & 325.6540 \\
& - & - & $(0.0112)$ & $(0.2591)$ & $(1.9119)$ & $(32.9011)$ \\
OLLMW & 0.0155 & 5.0157 & 4.4375 & 2.3343 & - & - \\
& $(0.0011)$ & $(0.0384)$ & $(0.2833)$ & $(0.9855)$ & - & - \\
KW & 30.0138 & 0.1988 & 0.0997 & 1.1214 & - & - \\
& $(13.2190)$ & $(0.0336)$ & $(0.6460)$ & $(0.7242)$ & - & - \\
BW & 8.4143 & 0.0660 & 2.1023 & 0.5458 & - & - \\
& $(4.7670)$ & $(0.0137)$ & $(0.2589)$ & $(0.0024)$ & - & - \\
\hline
\end{tabular}

Table 5. Values of the MLEs of the models parameters and their corresponding SEs (in parentheses) for Rainfall.

\begin{tabular}{ccccccc}
\hline Model & $\boldsymbol{\alpha}$ & $\boldsymbol{\beta}$ & $\boldsymbol{\mu}$ & $\boldsymbol{\theta}$ & $\boldsymbol{\lambda}$ & $\gamma$ \\
\hline REW & 0.9360 & 1.0760 & 0.2675 & 10.3239 & - & - \\
& $(0.8883)$ & $(1.2213)$ & $(0.6002)$ & $(1.5476)$ & - & - \\
TW & 9.9062 & 1.1254 & - & - & 0.3186 & - \\
& $(1.5148)$ & $(0.0591)$ & - & - & $(0.7906)$ & - \\
MOEW & - & - & 0.0623 & 10.0833 & 1.8004 & 238.9843 \\
& - & - & $((0.3398))$ & $(1.4870)$ & $(2.8477)$ & $(19.585)$ \\
OLLMW & 0.0192 & 0.5756 & 3.1829 & 3.2223 & - & - \\
& $(1.5733)$ & $(1.1585)$ & $(11.4127)$ & $(16.6993)$ & - & - \\
KW & 1.4830 & 0.1384 & 3.2070 & 9.3111 & - & - \\
& $(0.4889)$ & $(0.0301)$ & $(0.4722)$ & $(0.3949)$ & - & - \\
BW & 1.6104 & 5.1412 & 0.1601 & 7.1189 & - & - \\
& $(1.8937)$ & $(9.5075)$ & $(1.4302)$ & $(4.9144)$ & - & - \\
\hline
\end{tabular}

The rule is universal: a lower value of $(-\hat{\ell}), \mathrm{AIC}, \mathrm{W}, \mathrm{AD}$ and $\mathrm{KS}$, and a larger value of the $p$-value of the KS test, indicate a better fit. The obtained results for the considered models are given in Tables 6-8 for Carbon dioxide, Annual flood discharges and Rainfall, respectively. 
Table 6. Values of the $-\hat{\ell}$, akaike information criterion (AIC), BIC, W, AD, K-S with $p$-value of the considered models for Carbon dioxide.

\begin{tabular}{ccccccc}
\hline Model & $-\hat{\ell}$ & AIC & W & AD & KS & $p$-Value (KS) \\
\hline REW & 49.9510 & 107.9020 & 0.0368 & 0.2094 & 0.0670 & 0.9628 \\
TW & 51.2991 & 108.5983 & 0.0409 & 0.2381 & 0.0840 & 0.8239 \\
MOEW & 52.1708 & 112.3416 & 0.0695 & 0.4235 & 0.0912 & 0.7396 \\
OLLMW & 50.5856 & 109.1714 & 0.0398 & 0.2371 & 0.0831 & 0.8337 \\
KW & 50.3412 & 108.6825 & 0.0420 & 0.2427 & 0.0833 & 0.8315 \\
BW & 50.2656 & 108.5313 & 0.0434 & 0.2465 & 0.0726 & 0.9294 \\
\hline
\end{tabular}

Table 7. Values of the $-\hat{\ell}$, AIC, BIC, W, AD, K-S with $p$-value of the considered models for Annual flood discharges.

\begin{tabular}{ccccccc}
\hline Model & $-\hat{\ell}$ & AIC & W & AD & KS & $p$-Value (KS) \\
\hline REW & 215.6351 & 439.2702 & 0.0291 & 0.2103 & 0.0603 & 0.9949 \\
TW & 224.0996 & 454.1992 & 0.3258 & 2.0077 & 0.4460 & 0.0001 \\
MOEW & 220.9355 & 449.8709 & 0.1290 & 0.8376 & 0.1010 & 0.7117 \\
OLLMW & 220.5530 & 449.1061 & 0.1432 & 0.9322 & 0.0981 & 0.7439 \\
KW & 216.1038 & 440.2075 & 0.0490 & 0.3455 & 0.0816 & 0.9061 \\
BW & 253.2677 & 514.5354 & 0.1119 & 0.7453 & 0.4163 & 0.0000 \\
\hline
\end{tabular}

Table 8. Values of the $-\hat{\ell}$, AIC, BIC, W, AD, K-S with $p$-value of the considered models for Rainfall.

\begin{tabular}{ccccccc}
\hline Model & $-\hat{\ell}$ & AIC & W & AD & KS & $p$-Value (KS) \\
\hline REW & -22.5251 & -37.0503 & 0.0188 & 0.1269 & 0.0662 & 0.9979 \\
TW & -21.4078 & -36.8156 & 0.0336 & 0.2022 & 0.2075 & 0.0980 \\
MOEW & -21.6826 & -35.3651 & 0.0317 & 0.2012 & 0.0808 & 0.9762 \\
OLLMW & -21.6770 & -35.3497 & 0.0242 & 0.1794 & 0.0751 & 0.9891 \\
KW & -22.4097 & -36.8195 & 0.0207 & 0.1366 & 0.0846 & 0.9635 \\
BW & -22.4359 & -36.8718 & 0.0198 & 0.1326 & 0.0687 & 0.9914 \\
\hline
\end{tabular}

For the three data sets, Tables 6-8 indicate that the REW model has the smallest value for $-\hat{\ell}$, $\mathrm{AIC}, \mathrm{BIC}, \mathrm{W}$, AS and K-S, and the largest value of the $p$-value for the KS test (which is near optimal in each case, in the sense that $p$-value $\approx 1$ ), indicating that it provides the best fits among those of the concurrence.

We illustrate this sharpness but plotting various fits of the REW model, as the curves of the fitted pdfs, cdfs and sfs, i.e., $\hat{f}(x)=f(x ; \hat{\alpha}, \hat{\beta}, \hat{\mu}, \hat{\theta}), \hat{F}(x)=F(x ; \hat{\alpha}, \hat{\beta}, \hat{\mu}, \hat{\theta})$ and $\hat{S}(x)=$ $S(x ; \hat{\alpha}, \hat{\beta}, \hat{\mu}, \hat{\theta})$, over the corresponding histograms, empirical cdfs and empirical sfs, respectively, and the probability-probability (P-P) plots in Figures 3-5 for Carbon dioxide, Annual flood discharges and Rainfall, respectively. 

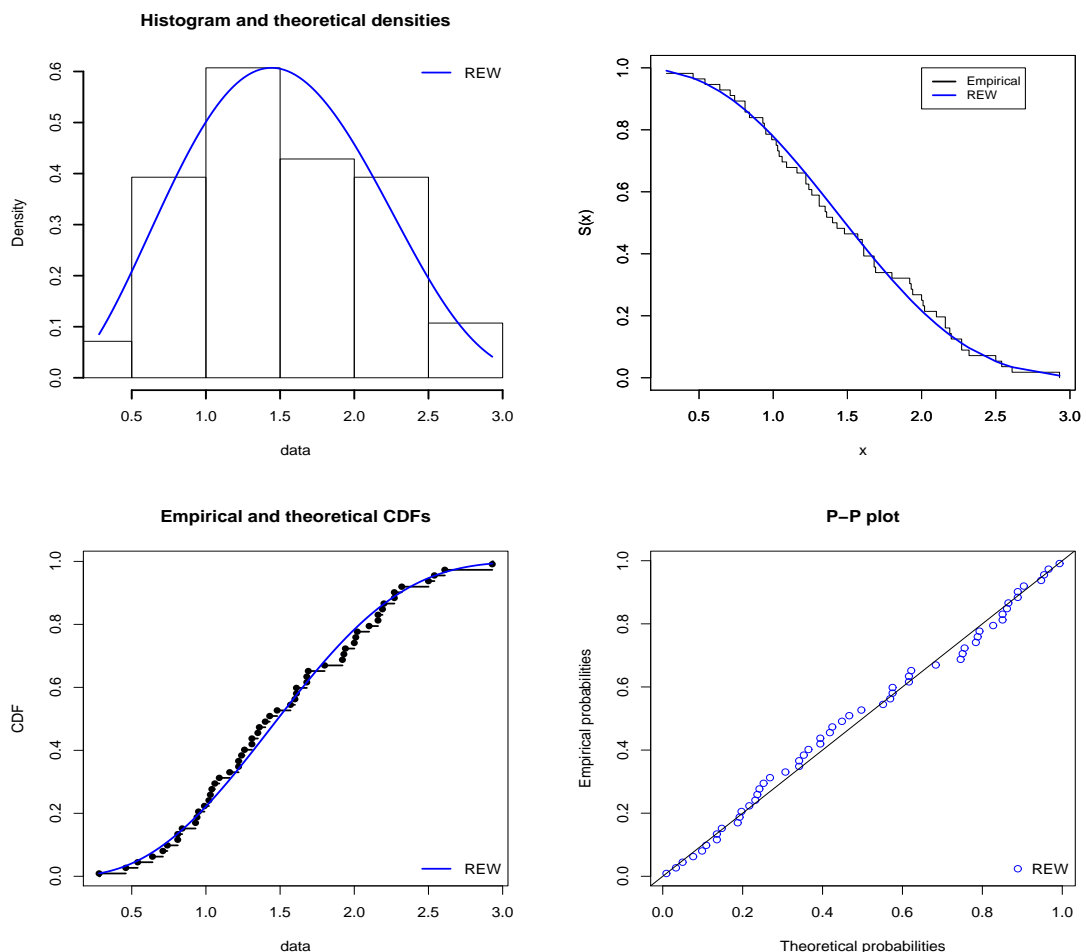

Figure 3. Plots of various estimated fits of the REW model (estimated pdfs, sfs, cdfs and P-P plots) for Carbon dioxide.
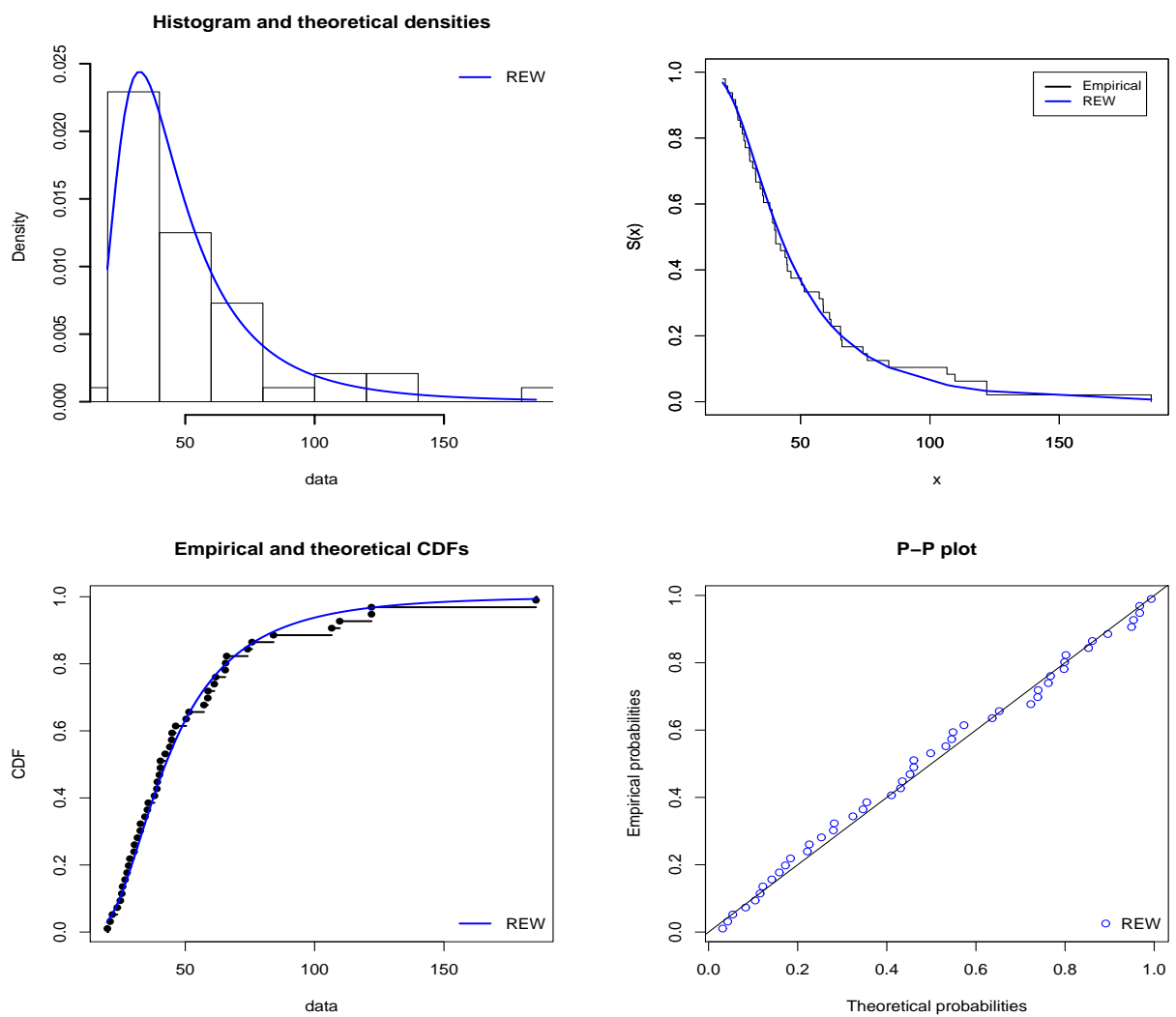

Figure 4. Plots of various estimated fits of the REW model (estimated pdfs, sfs, cdfs and P-P plots) for Annual flood discharges. 

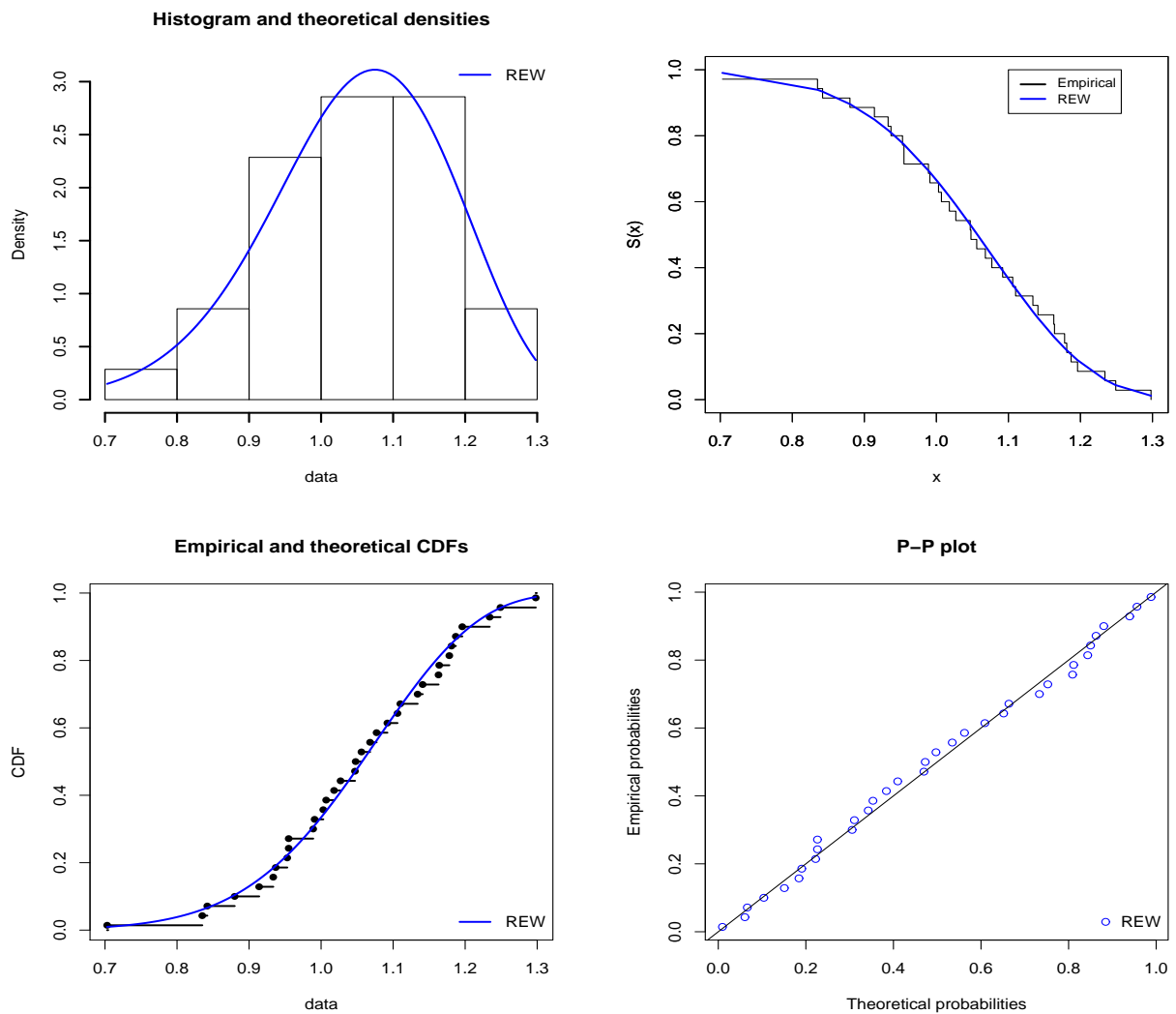

Figure 5. Plots of various estimated fits of the REW model (estimated pdfs, sfs, cdfs and P-P plots) for Rainfall.

In all the visual investigations of Figures 3-5, near perfect fits are observed, attesting the adequateness of the REW model for further studies on these data sets, among others.

\section{Concluding Remarks}

The paper started by providing a new theoretical result, involving a generalized cumulative distribution function, which can be viewed as an extension of the one defining the TIIHL-G family. It is called the ratio exponentiated-generated (RE-G) family. Then, we used this new result to elaborate upon a new attractive flexible family of distributions from the statistical point of view. In particular, a new modified four-parameter Weibull distribution is derived, called the REW distribution. We show how it can be applied in a concrete statistical framework, involving the analysis of data sets with different features. In particular, it is proved that the REW model can perform quite better than the following five other well-reputed models: TW, MOEW, OLLMW, KW and BW models, all having a plethora of applications in various articles. The same fate is expected for the REW model. As a perspective of work following the same scheme, since the RE-G and MO-G families are complementary in their definitions, one can consider a generalization of the MO-G family by providing an answer to the following question: What are the possible values for $\alpha, \beta$ and $\theta$ such that the following function has the properties of a cdf?

$$
F(x ; \alpha, \beta, \theta, \xi)=1-\frac{\theta\left[1-G(x ; \xi)^{\beta}\right]}{1-(1-\theta)\left[1-G(x ; \xi)^{\alpha}\right]}, \quad x \in \mathbb{R} .
$$

This expression is motivated by the following facts: for $\alpha=\beta=1, F(x ; \alpha, \beta, \theta, \xi)$ becomes the cdf of the MO-G family (not covered by the RE-G family), and, on the other side, by taking $\theta=1 / 2$, we get $F(x ; \alpha, \beta, \theta, \xi)=\left[G(x ; \xi)^{\alpha}+G(x ; \xi)^{\beta}\right] /\left[1+G(x ; \xi)^{\alpha}\right]$, corresponding to the cdf of the M-G family by [11] with two baseline exponentiated cdfs with power parameters $\alpha$ and $\beta$. This perspective 
thus unifies this two families, generating a myriad of new ratio-type distributions with possible wide range of values for $\alpha, \beta$ and $\theta$, beyond the standard positive values. The complete answer remains a mathematical challenge, needing deep investigations in the future.

Author Contributions: R.A.R.B., F.J., C.C. and M.E. have contributed equally to this work. All authors have read and agreed to the published version of the manuscript.

Funding: This work was funded by the Deanship of Scientific Research (DSR), King AbdulAziz University, Jeddah, under grant No. (RG-2-150-37).

Acknowledgments: We warmly thank the three reviewers for their thorough and constructive comments. This project was funded by the Deanship of Scientific Research (DSR), at King Abdulaziz University, Jeddah, under grant No. (RG-2-150-37). The authors, therefore, acknowledge with thanks to DSR technical and financial support.

Conflicts of Interest: The authors declare no conflict of interest.

\section{Appendix A}

The elements of the score function described in Section 3.6 are given by

$$
\begin{gathered}
\frac{\partial \ell(\alpha, \beta, \xi)}{\partial \alpha}=-\sum_{i=1}^{n} \frac{G\left(x_{i} ; \xi\right)^{\alpha}\left\{(\alpha-\beta) \log \left[G\left(x_{i} ; \xi\right)\right]+1\right\}}{\beta+(\beta-\alpha) G\left(x_{i} ; \xi\right)^{\alpha}}-2 \sum_{i=1}^{n} \frac{G\left(x_{i} ; \xi\right)^{\alpha} \log \left[G\left(x_{i} ; \xi\right)\right]}{1+G\left(x_{i} ; \xi\right)^{\alpha}}, \\
\frac{\partial \ell(\alpha, \beta, \xi)}{\partial \beta}=\sum_{i=1}^{n} \log \left[G\left(x_{i} ; \xi\right)\right]+\sum_{i=1}^{n} \frac{1+G\left(x_{i} ; \xi\right)^{\alpha}}{\beta+(\beta-\alpha) G\left(x_{i} ; \xi\right)^{\alpha}}
\end{gathered}
$$

and

$$
\begin{aligned}
\frac{\partial \ell(\alpha, \beta, \xi)}{\partial \xi} & =(\beta-1) \sum_{i=1}^{n} \frac{G\left(x_{i} ; \xi\right) \xi}{G\left(x_{i} ; \xi\right)}+\sum_{i=1}^{n} \frac{g\left(x_{i} ; \xi\right) \xi}{g\left(x_{i} ; \xi\right)}+\alpha(\beta-\alpha) \sum_{i=1}^{n} \frac{G\left(x_{i} ; \xi\right) \xi G\left(x_{i} ; \xi\right)^{\alpha-1}}{\beta+(\beta-\alpha) G\left(x_{i} ; \xi\right)^{\alpha}} \\
& -2 \alpha \sum_{i=1}^{n} \frac{G\left(x_{i} ; \xi\right) \xi G\left(x_{i} ; \xi\right)^{\alpha-1}}{1+G\left(x_{i} ; \xi\right)^{\alpha}},
\end{aligned}
$$

where $G\left(x_{i} ; \xi\right) \xi$ denotes the derivative of $G\left(x_{i} ; \xi\right)$ with respect to $\xi$, the same for $g\left(x_{i} ; \xi\right) \xi$.

\section{References}

1. Azzalini, A. A class of distributions which includes the normal ones. Scand. J. Stat. 1985, 12, 171-178.

2. Gupta, R.D.; Kundu, D. Exponentiated exponential family: an alternative to Gamma and Weibull distributions. Biom. J. 2001, 43, 117-130. [CrossRef]

3. Marshall, A.; Olkin, I. A new method for adding a parameter to a family of distributions with applications to the exponential and Weibull families. Biometrika 1997, 84, 641-652. [CrossRef]

4. Jones, M. Families of distributions arising from distributions of order statistics. Test 2004, 13, 1-43. [CrossRef]

5. Jones, M.; Pewsey, A. Sinh-arcsinh distributions. Biometrika 2009, 96, 761-780. [CrossRef]

6. Eugene, N.; Lee, C.; Famoye, F. Beta-normal distribution and its applications. Commun. Statistics-Theory Methods 2002, 31, 497-512. [CrossRef]

7. Shaw, W.T.; Buckley, I.R. The Alchemy of Probability Distributions: beyond Gram-Charlier Expansions, and a Skew-kurtotic-normal Distribution from a Rank Transmutation Map. arXiv 2009, arXiv:0901.0434.

8. Zografos, K.; Balakrishnan, N. On families of beta- and generalized gamma-generated distributions and associated inference. Stat. Methodol. 2009, 6, 344-362. [CrossRef]

9. Gómez-Déniz, E.; Calderín, E. Modelling insurance data with the Pareto ArcTan distribution. ASTIN Bull. 2015, 45, 639-660. [CrossRef]

10. Hassan, A.S.; Elgarhy, M.; Shakil, M. Type II Half Logistic family of distributions with applications. Pak. J. Stat. Oper. Res. 2017, 2, 245-264.

11. Kumar, D.; Singh, U.; Singh, S.K.; Mukherjee, S. The new probability distribution: An aspect to a Life time distribution. Math. Sci. Lett. 2017, 6, 35-42. [CrossRef] 
12. Klein, J.P.; Moeschberger, M.L. Survival Analysis: Techniques for Censored and Truncated Data, 2nd ed.; Springer: New York, NY, USA, 2003.

13. MacGillivray, H.L. Skewness and Asymmetry: Measures and Orderings. Ann. Stat. 1986, 14, $994-1011$. [CrossRef]

14. Cordeiro, G.M.; de Castro, M. A new family of generalized distributions. J. Stat. Comput. Simul. 2011, 81, 883-893. [CrossRef]

15. Cordeiro, G.M.; Ortega, E.M.; Nadarajah, S. The Kumaraswamy Weibull distribution. J. Frankl. Inst. 2010, 347, 1399-1429. [CrossRef]

16. Casella, G.; Berger, R.L. Statistical Inference; Duxbury Advanced Series Thomson Learning: Pacific Grove, CA, USA, 2002.

17. Ghitany, M.E.; Gómez-Déniz, E.; Nadarajah, S. A new generalization of the Pareto distribution and its application to insurance data. J. Risk Financ. Manag. 2018, 11, 10. [CrossRef]

18. R Development Core Team. R: A Language and Environment for Statistical Computing; R Foundation for Statistical Computing: Vienna, Austria, 2009.

19. Marinho, P.R.D.; Silva, R.B.; Bourguignon, M.; Cordeiro, G.M.; Nadarajah, S. AdequacyModel: An R package for probability distributions and general purpose optimization. PLoS ONE 2019, 14, e0221487. [CrossRef]

20. Gupta, R.D.; Kundu, D. A new class of weighted exponential distributions. Statistics 2009, 43, 621-634. [CrossRef]

21. Khaleel, M.A.; Ibrahim, N.A.; Shitan, M.; Merovci, F.; Rehman, E. Beta burr type $\mathrm{x}$ with application to rainfall data. Malays. J. Math. Sci. 2017, 11, 73-86.

22. Aryal, G.R.; Tsokos, C.P. Transmuted Weibull distribution: A generalization of the Weibull probability distribution. Eur. J. Pure Appl. Math. 2011, 4, 89-102.

23. Pogány, T.K.; Saboor, A.; Provost, S. The Marshall-Olkin exponential Weibull distribution. Hacet. J. Math. Stat. 2015, 44, 1579-1594. [CrossRef]

24. Saboor, A.; Alizadeh, M.; Khan, M.N.; Gosh, I.; Cordeiro, G.M. Odd LogLogistic modified Weibull distribution. Mediterr. J. Math. 2017, 14, 96. [CrossRef]

25. Lee, C.; Famoye, F.; Olumolade, O. Beta-Weibull distribution: some properties and applications to censored data. J. Mod. Appl. Stat. Methods 2017, 6, 173-186. [CrossRef]

26. Chen, G.; Balakrishnan, N. A general purpose approximate goodness-of-fit test. J. Qual. Technol. 1995, 27, 154-161. [CrossRef]

27. Massey, F.J., Jr. The Kolmogorov-Smirnov test for goodness of fit. J. Am. Stat. Assoc. 1951, 46, 68-78. [CrossRef]

(C) 2020 by the authors. Licensee MDPI, Basel, Switzerland. This article is an open access article distributed under the terms and conditions of the Creative Commons Attribution (CC BY) license (http://creativecommons.org/licenses/by/4.0/). 